\title{
Computer vision on magnetic resonance images
}

\author{
Wido MENHARDT and Karl-Heinrich SCHMIDT \\ Philips GmbH Forschungslaboratorium Hamburg, Vogt-Kölln-Str. 30, D-2000 Hamburg, FRG
}

Received 30 June 1988

\begin{abstract}
We present an approach for the automated interpretation of transaxial cranial magnetic resonance images. After a brief outline of our notation and basic assumptions, the overall design consisting of a neurological inference engine, a set of image processing operators and a configurating component for these operators is presented.
\end{abstract}

Key words: Computer vision, magnetic resonance.

\section{Introduction}

The appearance of magnetic resonance images ('MR-images') depends on several machine parameters and a set of spatially varying tissue parameters. Some of these tissue parameters are the spin density $(\rho)$, the spin-lattice-relaxation time $\left(T_{1}\right)$ and the spin-spin-relaxation time $\left(T_{2}\right)$. Examples for machine parameters are the repetition time $\left(T_{\mathrm{R}}\right)$ or the echodelay time ( $\left.T_{\mathrm{E}}\right)$ (cf.[9]).

As an example, we give the signal function for a measurement called spin-echo sequence, which is approximately

$$
\begin{gathered}
S(x, y) \propto \rho(x, y) *\left(1-\exp \left(-T_{\mathrm{R}} / T_{1}(x, y)\right)\right) \\
* \exp \left(-T_{\mathrm{E}} / T_{2}(x, y)\right) .
\end{gathered}
$$

The values for the tissue parameters $\rho, T_{1}$ and $T_{2}$ can be derived for each voxel represented by a pixel from a set of measurements with different $T_{\mathrm{E}}$ and $T_{\mathrm{R}}$ values.

We can thus define the $(2+3)$-dimensional Euclidian space $M R_{2}$ which is generated by two spatial coordinates and three coordinates for the tissue parameters $\rho, T_{1}$ and $T_{2}$. For a fixed type of a MR measurement sequence and the set of all grey-level tomograms $\mathrm{T}$, we define the functions

$$
F_{\mathrm{r}}: \mathrm{MR}_{2} \rightarrow \mathrm{T},
$$

the index $t$ denoting a tupel composed of the name of the sequence and its characteristic machine parameters; the tomogram in Figure 9 is a value of the function $F_{t}$ with $t=$ (SPIN-ECHO, $T_{\mathrm{E}}=200$, $T_{\mathrm{R}}=1500$ ). With $F_{\rho}, F_{T_{1}}$ and $F_{T_{2}}$, we denote the functions which compute the $\rho-, T_{1^{-}}$and $T_{2}$-parameter images, respectively.

By an interpretation of (MR-) images we mean the labelling of pixelsets - image regions -- with anatomical names. This is achieved by recursive knowledge-based separation of pixel sets in $\mathrm{MR}_{2}$ applying methods from pattern recognition, image processing and artificial intelligence according to a prespecified plan. Thus the image interpretation problem is divided into a set of subproblems each of which is solved by an especially designed operator.

A control framework is responsible for the configuration of these operators. The configuration task consists of

- a planning phase in which the locations of anatomical or pathological structures defined by a neurological inference engine are considered as goals and a set of operators is selected to achieve these goals,

- and an execution phase in which these operators are activated and their results are evaluated. 


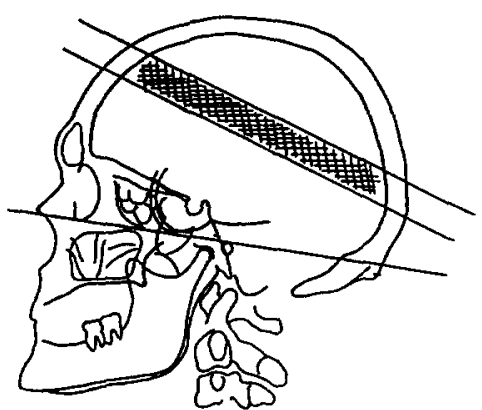

Figure 1. The part of the brain discussed in this paper.

Depending on this evaluation, operator parameters may be tuned to improve imperfect results. If this does not lead to satisfactory results, another planning phase is initiated to generate an alternative plan.

The whole system makes use of the two types of medical knowledge first described in [4]: one type is encoded textually in the primary description of pathologies' which defines and orders (objective) signs and (subjective) symptoms of the human body; the other type is encoded textually and pictorially in the 'secondary description of pathologies' which defines and orders normal and pathological units of the human body.

The architecture of the system reflects the clinical use of these two knowledge sources.

The characteristics of this system are:

(a) The evaluation of MR tomograms by a radiological expert is based on other data than computer vision in $\mathrm{MR}_{2}$ : Whereas the clinician uses only greylevel images, our computer system can utilize multispectral information in $\mathrm{MR}_{2}$ directly.

(b) The validity of an evaluation of a tomogram by a radiologist is based on attribution of individual competence. Radiology has not developed an operationalized method to discriminate correct and incorrect interpretations of medical images. Instead, we provide an operationalized approach.

(c) The language used for the description of MR- tomograms in radiology is based on terms whose determinance and uniformity cannot be discriminated from ordinary language. By incorporating the radiological terms into an evaluation of image processing operators their determinance and uniformity is increased [12].

The system has been developed for diseases located in the transaxial supraorbital slice of the brain shown in Figure 1.

\section{Methods}

\section{A. Neurological inference engine}

A neurological inference engine is designed for utilizing knowledge of the 'primary description of pathologies' to restrict possible locations of lesions (defined in the 'secondary description of pathologies').

Signs and symptoms are grouped according to the classes defined by the clinical symptomatology and represented as frames. Generally all frames representing knowledge about signs and symptoms contain:

(i) an attribute 'anatomical_location' containing a list of anatomical locations where the local sign or symptom may be caused;

(ii) an attribute 'neurological_evidence' which contains a list of pathologies the system knows to be a possible reason for the local sign or symptom;

(iii) an attribute 'status' which allows to declare whether the local sign or symptom is present for the patient under consideration.

Two frames belonging to two different symptoms are connected by an 'IS- $\mathrm{A}$ '-edge if and only if the intensional meaning of one (frame-)name comprises the intensional meaning of the other one; as an example the internal ordering of epileptical attacks is displayed in Figure 2.

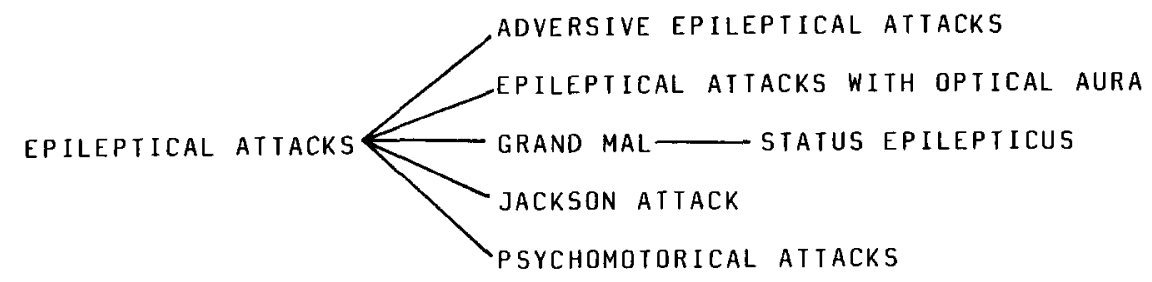

Figure 2. Part of the graphic display of the neurological knowledge base showing 'epileptical attacks'. 
Since the machine internal extension of a symptom, i.e. its presence and its markedness for a patient [15], has to be cvaluated interactively by a medical - user, the user interface contains panels which allow the assignment of values to the 'status'attribute by a simple mouse click; in Figure 3 such a panel is shown for the group of symptoms 'epileptical-attacks'.

Thus symptomatical profiles of patients are determined comfortably by establishing sentences of the type

"Patient $P$ has presumably symptom or sign $S$ "

as true or false.

Every symptomatical profile is converted automatically by an inference mechanism into a profile of possible locations of lesions.

The names of all locations are also related to frames which principally contain:

(i) an attribute 'evidence counter' where the symptomatical hints of evidence that a lesion may be present at this location are counted;

(ii) documented data about the normal $\rho$-, $T_{1}$ and $T_{2}$-distributions;

(iii) a list of all signs and symptoms the system knows to be indicative for a defect at this location.

The (anatomical) locations are ordered in a di-

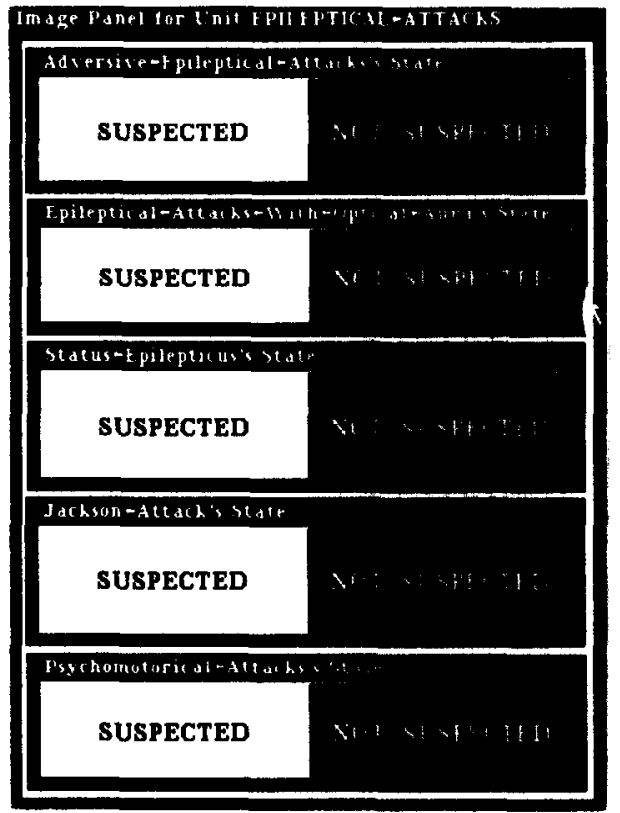

Figure 3. Active images for the symptoms shown in Figure 2.

rected AND/OR-graph $G=(N, E)$ (cf. Figure 4): Every element of the set of nodes $N$ is labelled with exactly one anatomical name or one of the two labels 'image' or 'background'; the set of edges $E=$ $\{[m, n] \mid m, n \in N\}$ is defined in the following way: starting with the root 'image' the labels of the successors have to denote

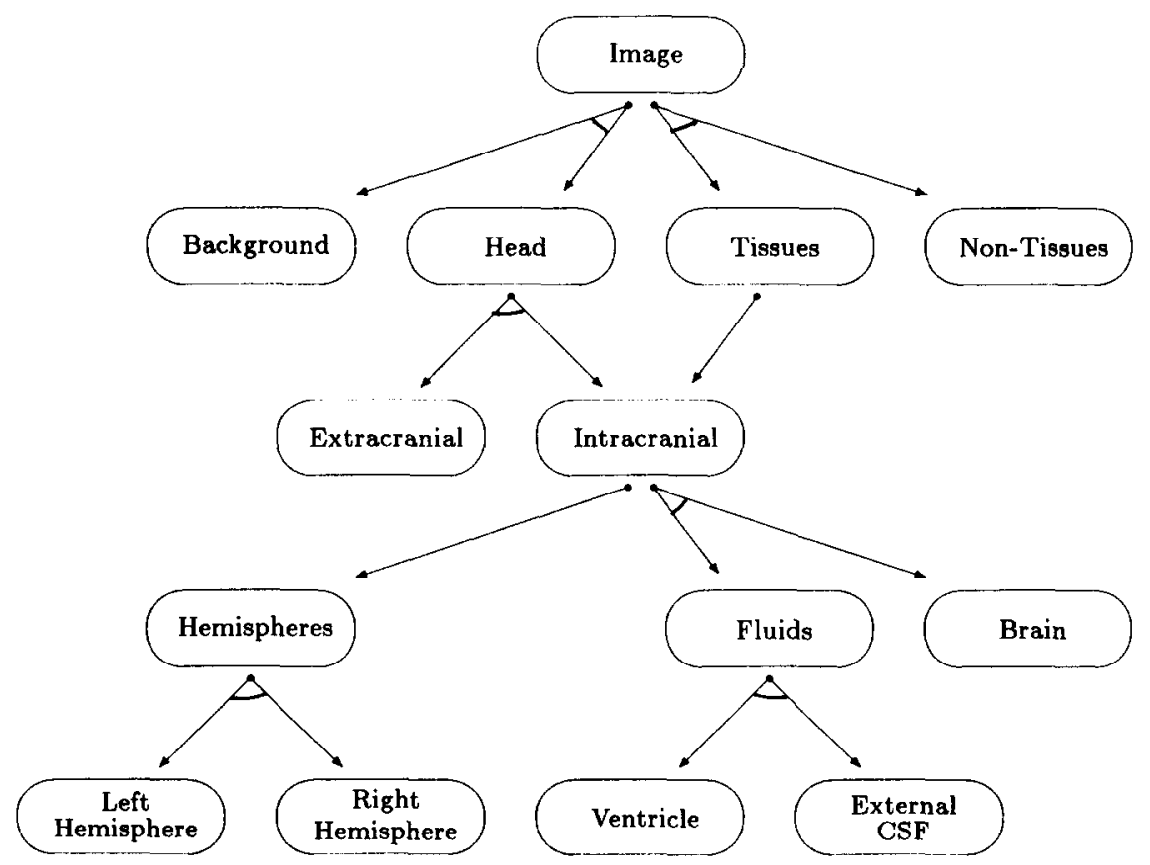

Figure 4. Part of the hierarchical ordering of anatomical locations. 
(a) an anatomical substructure of the structure denoted by the predecessor, e.g.

intracranial $\rightarrow$ fluids.

(b) a set of anatomical structures which form a complete partition of the structure denoted by the predecessor, e.g.

$$
\text { intracranial } \rightarrow \text { hemispheres. }
$$

The edges therefore define a 'HAS_PARTS'-relation. For a node $m$, the set of successors

$$
\operatorname{succ}(m)=\left\{n_{1}, n_{2}, n_{3}, \ldots . n_{t}\right\}
$$

is conjoined by an AND if and only if $n_{1}, n_{2}, \ldots, n_{t}$ form a complete (anatomical) partition of $m$. A subtree of the first five levels of $G$ is shown in Figure 4.

For a patient all nodes of type (a) with a positive entry in the attribute 'evidence counter' are defined as goals for a configurator of image interpretation operators (cf. B and C).

The interface between the neurological inference engine just described and the user is shown in Figure 5 .

\section{B. Interpretation operators}

Based on the definition of the graph $G=(N, E)$ (cf. A) an operator $O$ is defined for a set of (anatomical) names $N$ as a function

$$
\begin{aligned}
O: \operatorname{MR}_{2} \times N & \rightarrow \operatorname{MR}_{2} \times N \\
(p, m) & \mapsto(p, n), \quad n \in\{m\} \cup \operatorname{succ}(m) .
\end{aligned}
$$

In Figure 4, the names 'intracranial' and 'extracranial' e.g. denote a complete partition of 'head'.

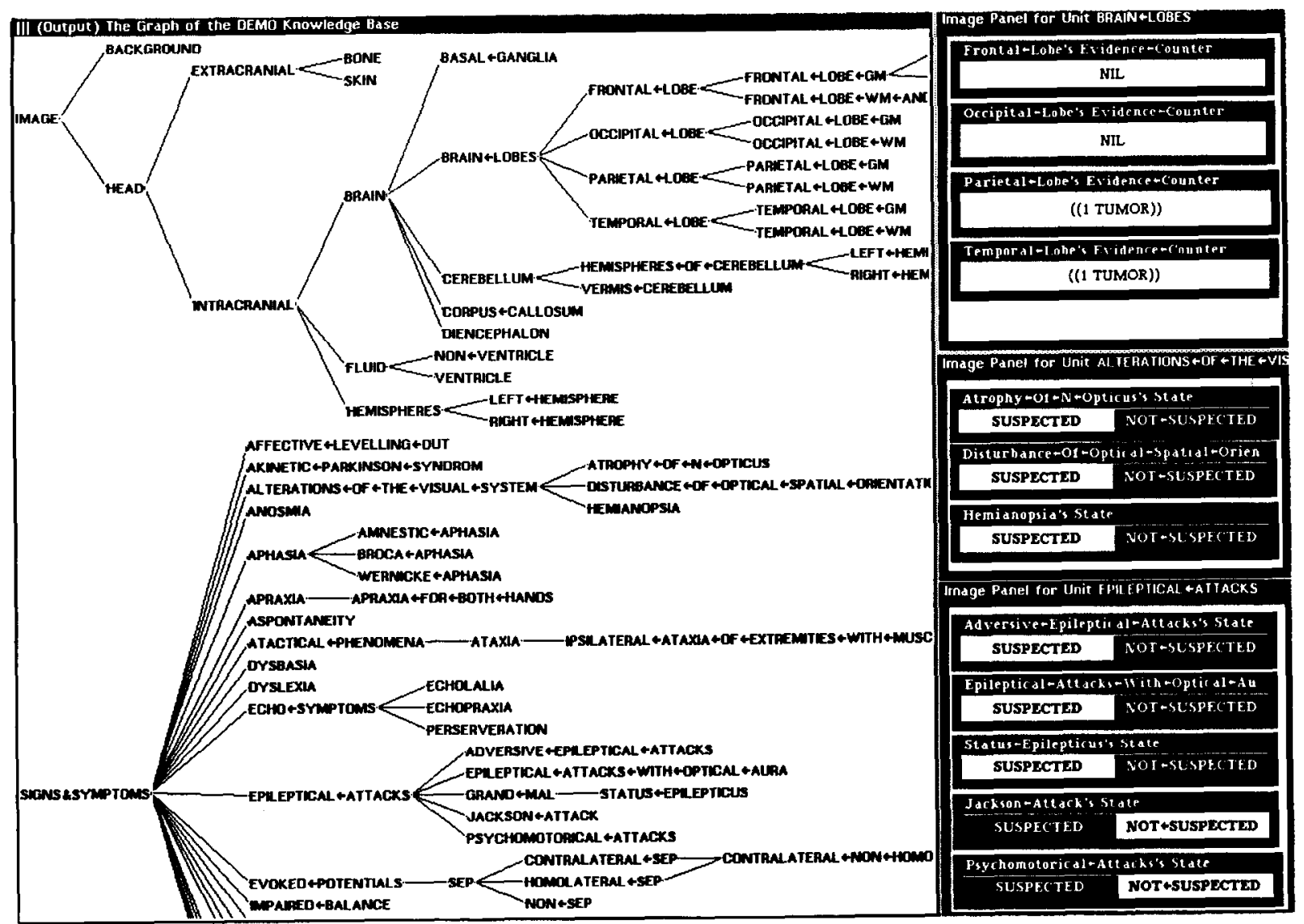

Figure 5. On this display it is possible to assign the values 'suspected' and 'not_suspected' to the attribute 'status' of the group of symptoms 'epileptical_attacks' and 'alterations_of_the visual_system' by means of active windows. Here the symptoms 'Jackson_attack' and 'psychomotorical_attack' give one hint for the presence of a tumor at the parietal and frontal lobe of the brain; this is indicated in the upper part of the display by another active window showing the values of the respective evidence counters. 
An operator constructed to realize this partition in $\mathrm{MR}_{2}$ is the identity on

$$
\left\{(p, \text { BACKGROUND }) \mid p \in \mathrm{MR}_{2}\right\}
$$

and has the values

$$
\{(p, \text { INTRACRANIAL }),(p, \text { EXTRACRANIAL })\}
$$

on

$$
\left\{(p, \mathrm{HEAD}) \mid p \in \mathrm{MR}_{2}\right\} .
$$

We exemplify these definitions for transaxial MR-images through the ventricular system (cf. Figure 1) by means of a detailed description of four basic operators: FInD_HeAd, FInD_InTRACRANiAL, Find_Fluids and FInD_Ventricles. As shown in Figure 4, these operators introduce a set of (anatomical) names, the unique root ('image') being the only predefined one.

\section{B.1. Operator FInD_HEAD}

In this section an operator realizing the partition

$$
\begin{aligned}
& \left\{(p, \text { IMAGE }) \mid p \in \mathrm{MR}_{2}\right\} \rightarrow \\
& \quad\left\{(p, \mathrm{BACKGROUND}) \mid p \in \mathrm{MR}_{2}\right\} \\
& \dot{\cup}\left\{(p, \text { HEAD }) \mid p \in \mathrm{MR}_{2}\right\}
\end{aligned}
$$

is described.

At an intact skull, the outmost anatomical structure of the head is skin. In contrast to calcifications, bone structures and background, the signal intensity of skin is rather high in $F_{\rho}$ (cf. Figure 6).

Furthermore, the contour of the head in the slice we examine resembles an ellipse. Since the regions with low values in $F_{\rho}$ within the head are rather small, this slice of the head can be modelled as an ellipse-shaped disk.

This leads to the generation of an elliptical coordinate system for these tomograms in the following way (Figure 6): The center of the coordinate system is calculated in $F_{\rho}$ by regarding all image points as points of a solid object with a mass proportional to its spin density. The calculation of the center of gravity of this object yields the center. In a similar way, the base vectors are calculated by means of the covariance matrix: The eigenvectors of this matrix multiplied with the corresponding eigenvalues form the desired base of the coordinate system. This is equivalent to the calculation of the axis and moments of inertia (cf. [13])

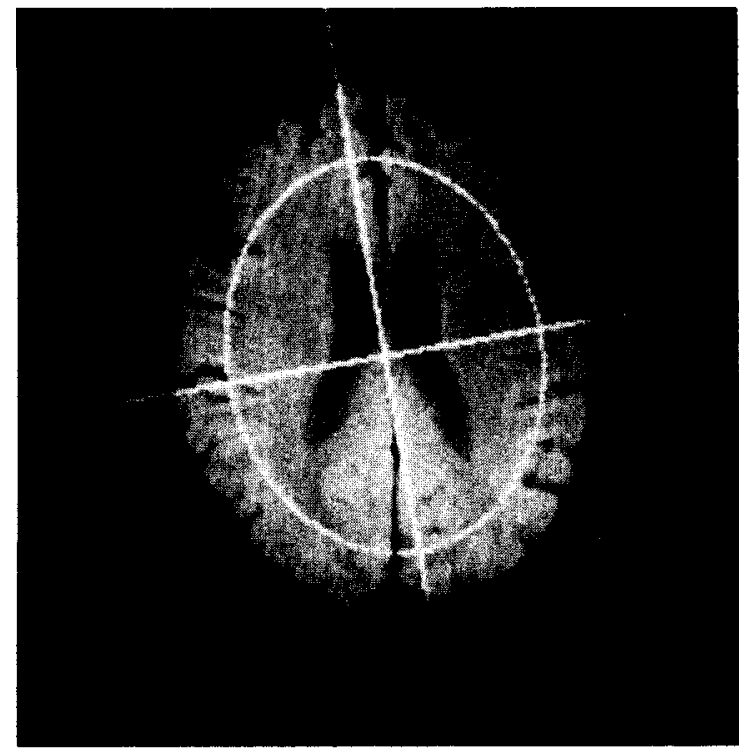

Figure 6. Spin density image with overlayed elliptical coordinate systems.

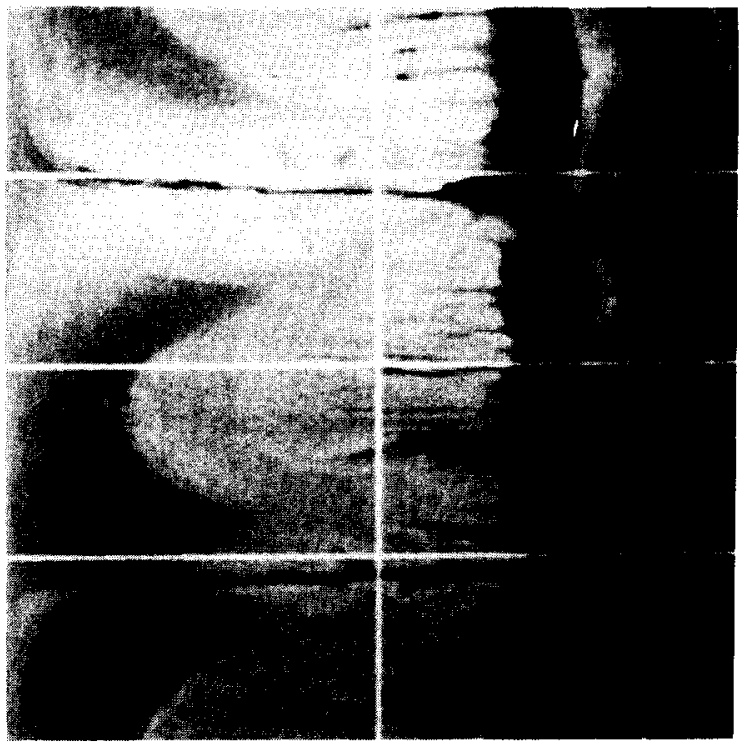

Figure 7. Transformed spin density image ( $r$ : horizontal, $\varphi$ : vertical) with an intermediary solution for the head contour after four iterations.

A representation of our image in these coordinates shows a spherical disk-like structure. A subsequent $(r, \varphi)$-polar-coordinate transformation yields an image where the contour of the head approximately forms a straight line paraliel to the $\varphi$-axis (Figure 7).

In this representation, generated by two coordinate transformations, we detect the head contour in 

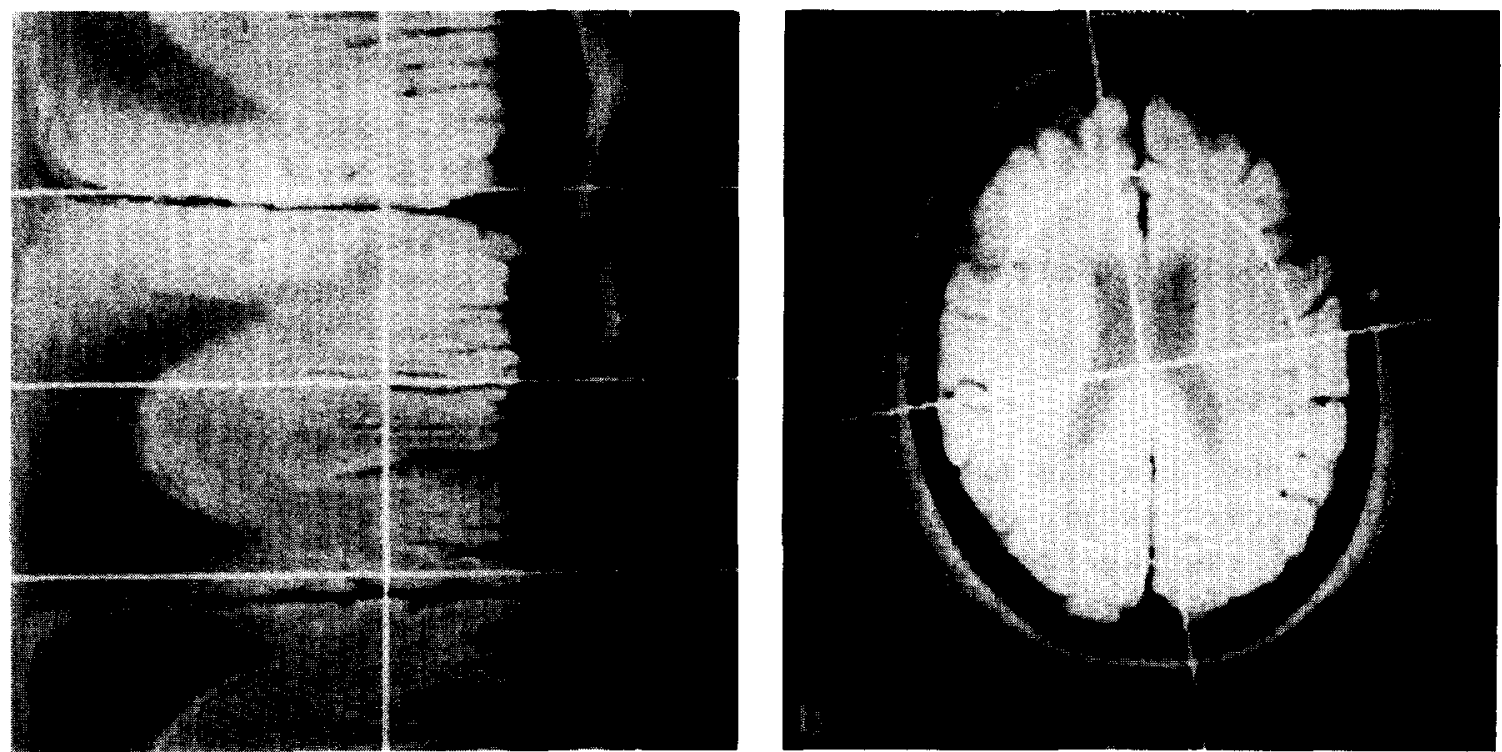

Figure 8. (a) Solution for head contour in transformed spin density image. (b) Backtransformed version of (a).

the following way. First, in the corners of the original image a mean spin density of the background - determined by noise - is calculated. As an initial contour we take a straight line with $r(\varphi)=r_{\max }$. Subsequently, an iterative loop consisting of two steps is activated.

In the first step, the value $r(\varphi)$ of the contour is decremented as long as the corresponding value of spin density is lower than the mean background spin density value. Because of background noise these values of $r(\varphi)$ will differ for different values of $\varphi$.

In a second step, each value of $r(\varphi)$ is replaced by the mean $r(\varphi)$ value in a specified window around the respective $\varphi$-value of the contour (Figure 7).

The iteration terminates, if there are no changes between two steps (Figures 8a, 8b).

\section{B.2. Operator FIND_INTRACRANIAL}

In this section an operator realizing the partition
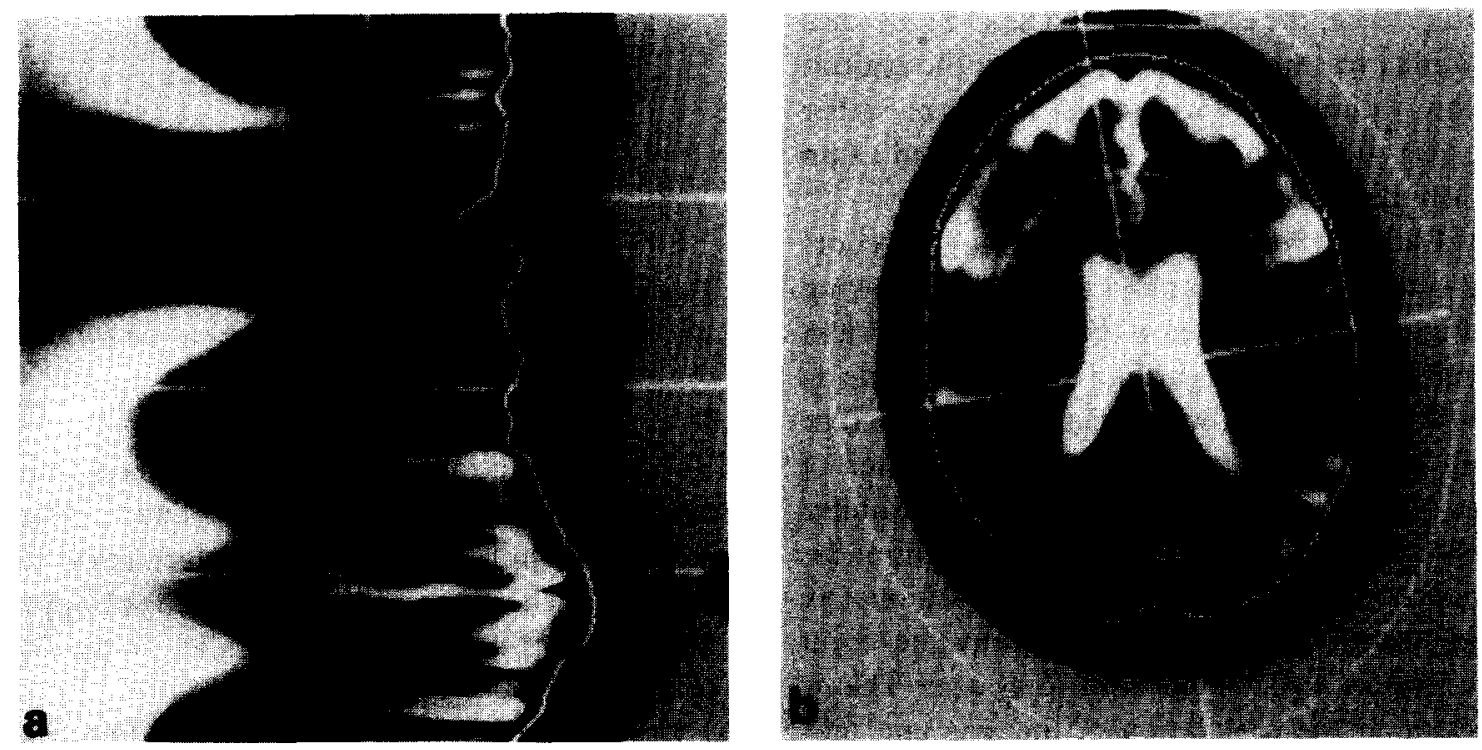

Figure 9. (a) Solution for the head contour in transformed spin-echo image. (b) Backtransformed version of (a). 


$$
\begin{aligned}
& \left\{(p, \text { HeAD }) \mid p \in \mathrm{MR}_{2}\right) \rightarrow \\
& \quad\left\{(p, \text { INTRACRANIAL }) \mid p \in \mathrm{MR}_{2}\right\} \\
& \quad \dot{\cup}\left\{(p, \text { EXTRACRANIAL }) \mid p \in \mathrm{MR}_{2}\right\}
\end{aligned}
$$

is described.

To detect the intracranial region of the head in transaxial tomograms, we first use the head contour yielded by the operator FIND_HEAD. We assume that the contour of the intracranial region is approximately parallel to the contour of the head for an intact skull. Furthermore, we know that bone structures have relatively low values in $F_{\rho}$ compared with the intracranial region. Additionally, bone is the only structure in the head with an ellipse-like shape and low spin density.

This information is used by shifting the head contour towards smaller $r$ until the sum of spin density along the contour is minimal. This starting contour lies entirely within the bone. To find the intracranial contour, the same iterative procedure as described in B.1 is applied (cf. Figure 9).

\section{B.3. Operator FIND_FLUIDS}

In this section an operator realizing the partition

$$
\begin{gathered}
\left\{(p, \text { INTRaCRaniaL }) \mid p \in \mathrm{MR}_{2}\right\} \rightarrow \\
\left\{(p, \text { FluIDS }) \mid p \in \mathrm{MR}_{2}\right\} \\
\dot{U}\left\{(p, \text { BRaIN }) \mid p \in \mathrm{MR}_{2}\right\}
\end{gathered}
$$

is specified.

The (cerebrospinal) fluid (CSF) has a very high intensity in $F_{T_{2}}$ (Figure 10a). Moreover, a histo-
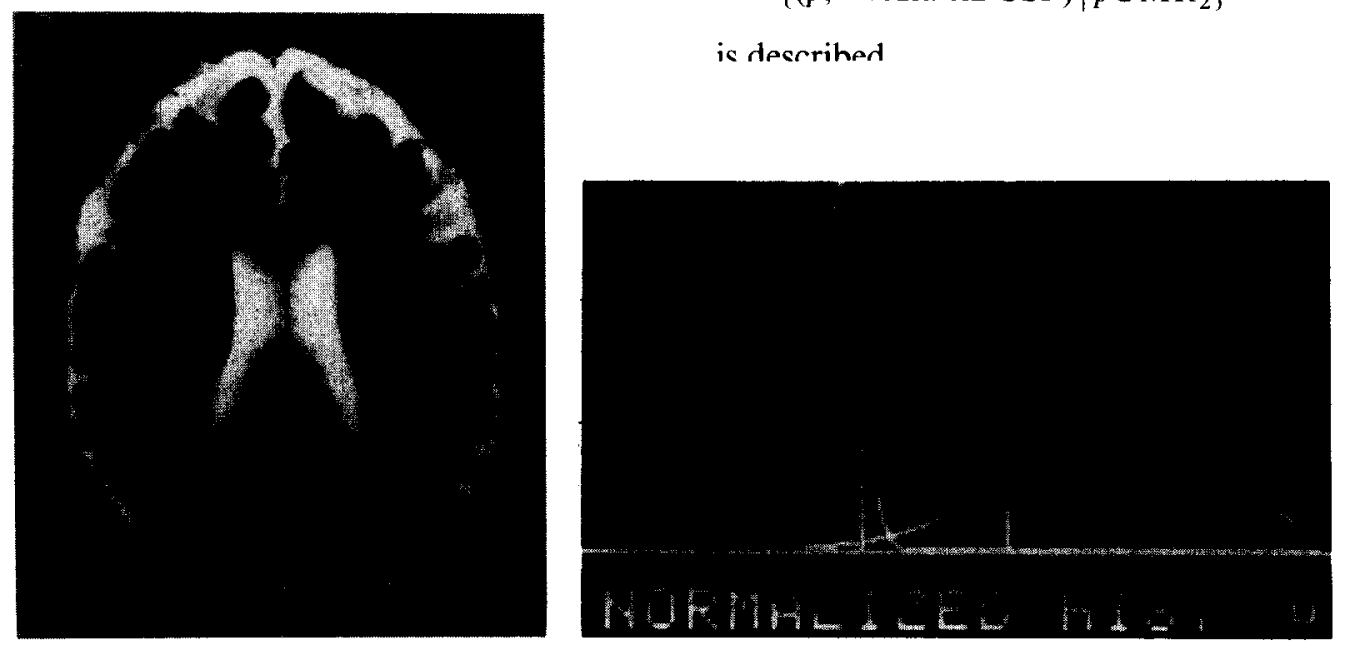

Figure 10. (a) $T_{2}$-image of intracranial region. (b) Modified $T_{2}$-histogram of intracranial region.

gram analysis of the $T_{2}$-distribution in the intracranial regions shows two modi: a lower one caused by the brain matter, a higher one caused by CSF (Figure 10b).

An approximation of this $T_{2}$-distribution by a sum of two gaussian distributions yields a criterion for a partition of the intracranial region into fluid and brain regions (cf. [2]).

To reduce data complexity, this criterion is applied to a segmented image consisting of some hundred connected pixelsets with a homogeneous tissue parameter distribution in $\mathrm{MR}_{2}$ (Figure 11); these regions are generated by a cluster analysis in $\left\langle\rho, T_{1}, T_{2}\right\rangle$ as a subspace of $\mathrm{MR}_{2}$ together with a connectivity condition (cf. [2], [11]): Each pixel in a region is classified with a binary clustering algorithm starting with the whole image; all subregions smaller than a predefined size are eliminated; each subregion is recursively partitioned with the same algorithm until the number of subregions is one.

The decision whether an intracranial region is labelled as CSF or brain is made in the following way: For each region, the sum of all pixels belonging to the lower mode of the histogram is compared with the sum of pixels belonging to the upper mode; the greater number leads to an attribution of the respective label (Figure 12).

\section{B.4. Operator Find_VEnTRICLE}

In this section an operator realizing the partition

$$
\begin{aligned}
& \left\{(p, \text { Fluids }) \mid p \in \mathrm{MR}_{2}\right\} \rightarrow\left\{(p, \mathrm{VENTRICLE}) \mid p \in \mathrm{MR}_{2}\right\} \\
& \dot{\cup}\left\{(p, \text { External CSF }) \mid p \in \mathrm{MR}_{2}\right\}
\end{aligned}
$$

is decerihed 


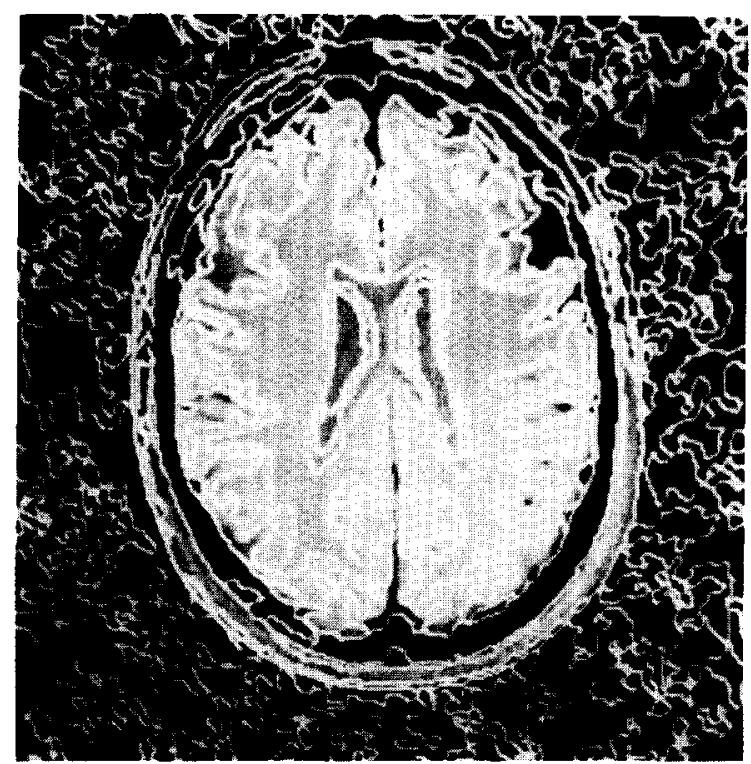

Figure 11. Spin density image with superimposed segment boundaries.

The ventricular system of transaxial slices of the brain consists of those central CSF regions which are not connected to external CSF regions in adjacent slices.

The information which has to be utilized for detection of the ventricular system is its central position in terms of the coordinate system defined above.

Thus groups of adjacent CSF regions are deter-

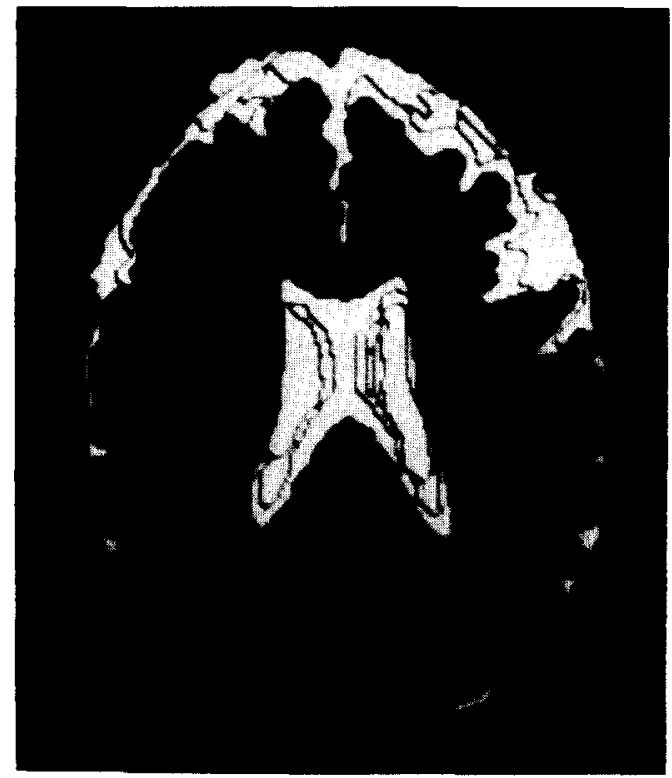

Figure 12. Regions interpreted as CSF (white) or brain (gray). mined and interpreted by a rule-based system containing 24 rules leading to small conflict sets and realizing the following semantics (cf. [3]): All groups which are adjacent to extracranial regions are excluded as candidates for the ventricular system. In the set of the remaining groups in each hemisphere, the one nearest to the center of gravity is interpreted as ventricular system; all other groups are labelled as external CSF (Figure 13).

An example for the rules used has the following logical shape:

If the present task is to collect ventricle candidates $\&$ there exists a region with name $\langle\mathrm{I}\rangle$

$\&$ with distance $\langle D\rangle$ to the center of the coordinate system

\& with position in hemisphere 〈HeMI >

\& with current interpretation 'FLuIDs'

$\&$ there does not exist a region with position in hemisphere〈HEMI >

$\&$ with interpretation FLUIDS

$\&$ with distance to center smaller than $\langle\mathrm{D}\rangle$

then generate a symbol

$\&$ bind it to a variable 〈ID >

$\&$ modify the current interpretation of $\langle\mathbf{I}\rangle$ to

'VENTRICLE-CANDIDATE'

$\&$ mark $\langle\mathrm{I}\rangle$ with $\langle\mathrm{ID}\rangle$

$\&$ start the task collect neighbours of $\langle$ ID $\rangle$

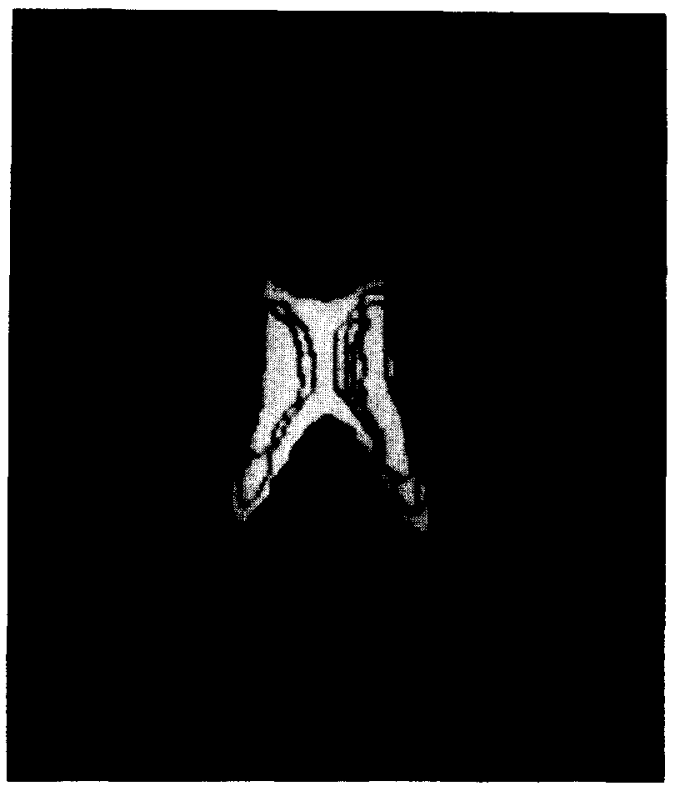

Figure 13. Regions interpreted as ventricular system (white) or external CSF (gray) 
In Ops5 that looks like:
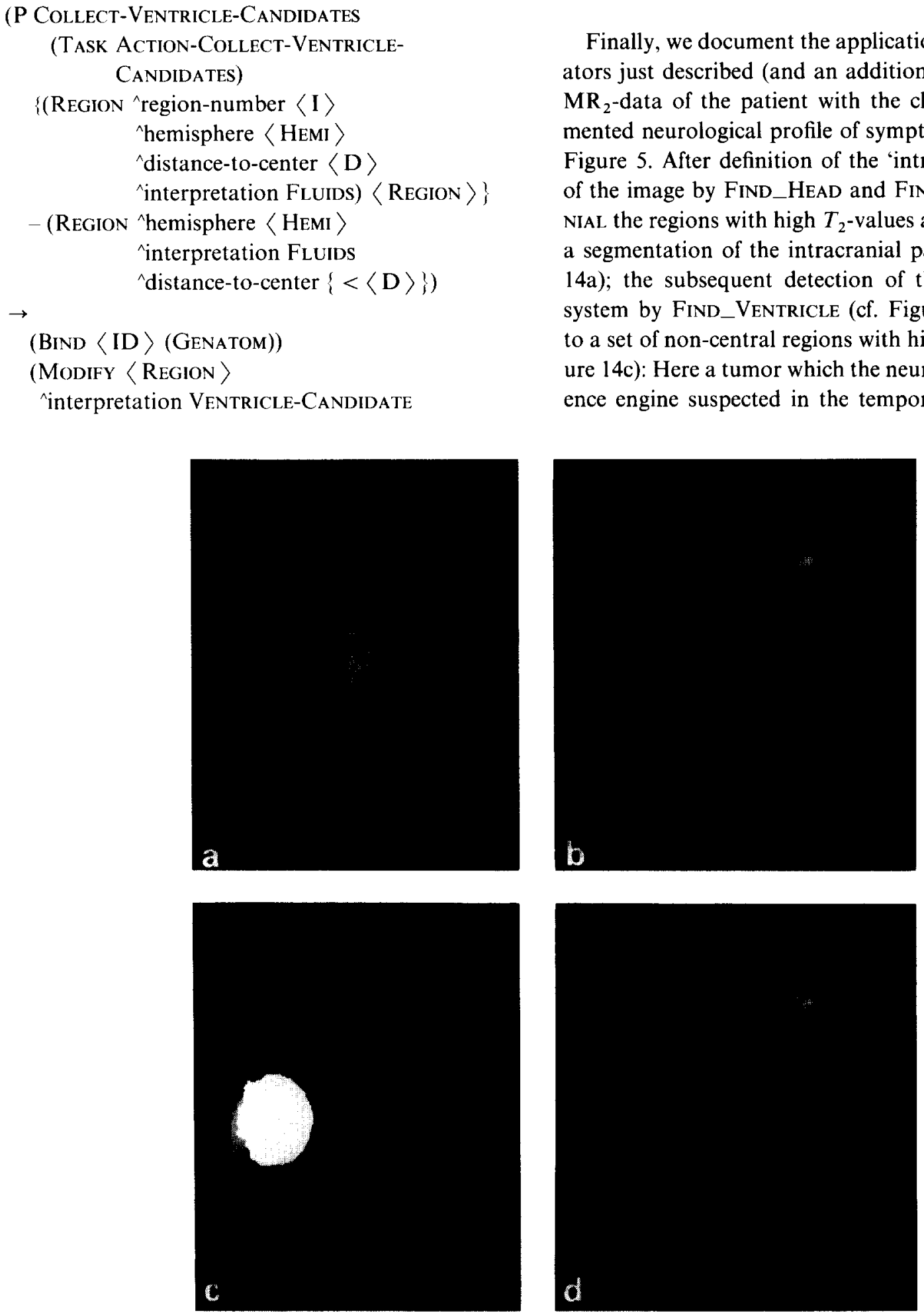

Figure 14. Detection of the ventricular system and a low-grade astrocytoma in a transaxial supraorbital MR-image.
${ }^{\wedge}$ id $\langle$ ID $\left.\rangle\right)$

(Make Task Collect Neighbours $\langle$ ID $\rangle)$ )

Finally, we document the application of the operators just described (and an additional one) to the $\mathrm{MR}_{2}$-data of the patient with the clinically documented neurological profile of symptoms shown in Figure 5. After definition of the 'intracranial' part of the image by FIND_HEAD and FIND_INTRACRANIAL the regions with high $T_{2}$-values are labelled on a segmentation of the intracranial part (cf. Figure 14a); the subsequent detection of the ventricular system by FIND_VENTRICLE (cf. Figure 14b) leads to a set of non-central regions with high $T_{2}$ (cf. Figure 14c): Here a tumor which the neurological inference engine suspected in the temporo-parietal left 
part of the brain is discriminated by an operator which detects large parameter-homogeneous components (cf. Figure 14d).

\section{Configurating system}

Principally, several ways for the decomposition of the interpretation task exist in the graph $G$; in Figure 4 you find e.g. two paths to the node 'INTRACRANIAL':

IMAGE $\rightarrow \mathrm{HEAD} \rightarrow$ INTRACRANIAL

or

IMAGE $\rightarrow$ TisSUES $\rightarrow$ INTRACRANIAL.

Therefore, different sequences of operators may be used to solve the interpretation task. Furthermore, it is possible to define different operators to solve the same problem using alternative methods.

This leads to a variety of possible sequences of operators for the solution of the interpretation problem. As a consequence, a control framework for the configuration of operators is required (cf. [7], $[8])$.

An expert (e.g. the designer) is able to compose the interpretation operators, tune their parameters and select alternative approaches in case of failure: He uses knowledge and experience about the performance of the operators under certain conditions and about their sensitivity to control parameters.

The ultimate goal is the elimination of the interaction between system and expert to achieve a fully automated interpretation of our MR-images. To do so, three topics have to be discussed with respect to configuration:

- knowledge representation,

- knowledge acquisition,

- inference.

\section{C.1. Knowledge representation}

Three general types of knowledge are involved in configurating interpretation operators. We will refer to them as technical, temporary and experienced knowledge.

Technical knowledge includes the definition of the required input data and parameters for each operator; furthermore, the input and output pixel sets are included which describe the specific problem an operator is designed for. Technical knowledge is static in the sense that it is never changed once an operator is integrated into the system.

82
Temporary knowledge is only generated and used for one case. It is categorized into three types:

- facts (e.c. transversal slice of head, measurement method),

- constraints (e.c. required speed and accuracy) and

- hypotheses (e.c. expected pathologies based on clinical findings). Examples for these 3 types are:

If 'VENTRICLE' IS-A-GOAL; an anatomical region then TAKe-Operator 'Find_Ventricle 9'; a specialized operator

because 'SLICE-9'; a radiological term: the slice of the brain shown in Figure 1

IS-A-FACT

If 'Find_Ventricle_9' is-Selected-Method; a selected operator

then SeT 'Minimal-Region-SizE': = 128; one of its parameters

because 'REASONABLE-TIME'; a constraint set before execution

IS-A-CONSTRAINT

If 'PATHOLOGY' IS-A-Goal.

then TAKE 'RS4_l'; an operator

because 'TEMPORAL-PARIETAL-TUMOR'; a location derived from the neurological inference engine IS-A-HYPOTHESIS

Based on these facts, constraints and hypotheses, a plan is generated. Such a plan is exactly one subgraph of $G$ the root of which is some element of $N$ (normally 'image') and whose terminal node is the goal. Arbitrary (anatomical) names in $N$ can be selected as goals (cf. A). It is also possible to define more than one goal leading to a tree-structured subgraph as a plan.

Temporary knowledge may be entered by the user at the start of the configurating process and during the process, as will be discussed below.

Experienced knowledge forms the kernel of the configurating knowledge. It represents reasons for the selection of operators and the setting and tuning of parameters. Experienced knowledge is acquired from the expert during the operation of the system. It is therefore stored in a dynamic knowledge base which will comprise and retained more knowledge after each run of the configuration system. 


\section{C.2. Knowledge acquisition}

Dynamic knowledge acquisition of experienced knowledge is essential since it is impossible to acquire and represent this kind of knowledge in advance. The expert can not formulate all his configurating knowledge by introspection. Therefore, a knowledge acquisition component is included to guide the decisions of the expert in certain situations and represent them in such a way that the system will be able to make the same decisions in similar situations.

This learning process is based on an easy concept: Each time a decision has to be made (operator selection, parameter setting, etc.), the system generates a proposal. If the expert does not agree with the proposal, he is given the opportunity to enter an alternative decision. He is then asked for a reason of his decision. He may enter his reason in one of the categories 'facts', 'constraints' and 'hypotheses'. If the reason is not in the temporary knowledge base yet, it is added then. The complete rule

\section{〈If goal then make-decision because reason 〉}

is added to the experienced knowledge base.

The next time the system encounters a similar goal, it will be able to make the same decision itself. The representation of the rule accentuates the final character of the if-clause and the causal character of the because-clause. The syntax stresses the different types of the two logical conditions of the rule in a way which is easier to understand for a non-expert than the logically equivalent formulation.

Although the configuration of a set of operators is based on a sequence of decisions which is believed to yield results optimal for radiological perception, these results can be unsatisfactory. This means that either the set of possible operators is incomplete or that the set of reasons describing the given situation is inadequate.

To exclude the second possibility of an imprecise description of the situation, the visual correctness of each intermediate result is controlled by an evaluation component. If the evaluation is positive, the execution can continue; if not, the user is prompted to evaluate the result in his terminology: a possible (negative) evaluation may be

"object: T attribute: contour value: too-fuzzy".
A vocabulary for possible attributes and values is presented to the user [12]. It contains terms from the radiological domain (cf. Table 1). Moreover, the user can add his own terms.

\section{C.3. Inference}

Inference is not limited to the retrieval of collected rules. For each goal there may (and will) be several rules. Only those rules with a because-clause matched by a predicate in the temporary knowledge base are activated. If more than one rule applies, a decision supported by the largest number of activated rules is made (or: proposed to the user).

This way of reasoning about decisions is strictly positive because it only takes support for each possible decision into account. This makes it rather difficult to exclude rules explicitely from being activated under special circumstances. Therefore, a negative type of rule is introduced which is established by asking the user for his reasons for not accepting one of the other possible decisions for a given goal. Rules of the form

\section{$\langle$ If goal then-don't take-decision because reason $>$,}

are generated and added to the experienced knowledge base.

Although a mechanism of negative support could be implemented using negative predicates in the first type of rules, this formulation more closely matches the human representation.

\section{Discussion}

The whole system reflects the clinical use of the knowledge described in (1): Starting with a neurological hypothesis, a tomogram of that part of the body which presumably contains a lesion is interpreted to evaluate the hypothesis using the primary (here: especially neurological) and secondary (here: especially radiological) descriptions of pathologies.

Radiological expertise is used in two ways: it guides the design of the operators and is used to evaluate their performance.

The attributes and values of the clinical observation language are exploited algorithmically to detect anatomical and pathological structures; the at- 
tributes of the clinical language used for the definition of the operators introduced in (2) are summarized in Table 1. The evaluation of the operators is done by the same attributes and values.

This approach offers the following advantages:

(1) Validation: By incorporating the radiological terms into the evaluation of the configuration process, the performance of the system can be validated in domain specific terms.

(2) Documentation: The picture description language of a radiological domain can be documented and refined; this allows competent judgements about images without continuous local presence of personal competence in a clinic.

(3) Consistency: The combination of radiological terms with the performance of operators allows checks of visual consistency in a radiological domain; thus, traditions can be substituted by operationalized methods.

The neurological inference engine has been implemented in KeE on a Symbolics Lisp Machine under GENERA. The interpretation operators are all written in Ops5 with PASCAL subprocedures for pixel level operations. They are operational on a VAX8600 under VMS. The configuration system is implemented on the same machine in OPS5. The execution of interpretation operators is controlled via VMS subprocesses. The Symbolics and VAX machines are linked via ETHER NET.

The system has been developed on textual and pictorial data of twenty patients suffering from tumors and multiple sclerosis.

\section{References}

[1] Ballard, D. and C. Brown (1982). Computer Vision, Prentice-Hall, Englewood Cliffs, NJ.

[2] Duda, R.O. and P.E. Hart (1973). Pattern Classification and Scene Analysis, Wiley, New York.

[3] Forgy, C.L. and J. McDermott (1977). OPS, A domain-independent production system language. Proc. Joint Conference on Artificial Intelligence, MIT, Cambridge, p. 933ff.

[4] Foucault, M. (1972). Naissance de la Clinique, Paris.

[5] Hempel. C.G. (1948). Studies in the logic of explanation, Philosophy of Science XV, p. 135ff.

[6] Menhardt, W. and K.-H. Schmidt (1987). Automated interpretation of transaxial MR-images of the brain. Proc. of Computer Assisted Radiology, CAR'87, Berlin, Springer, $\mathrm{p}$. $386 \mathrm{ff}$.

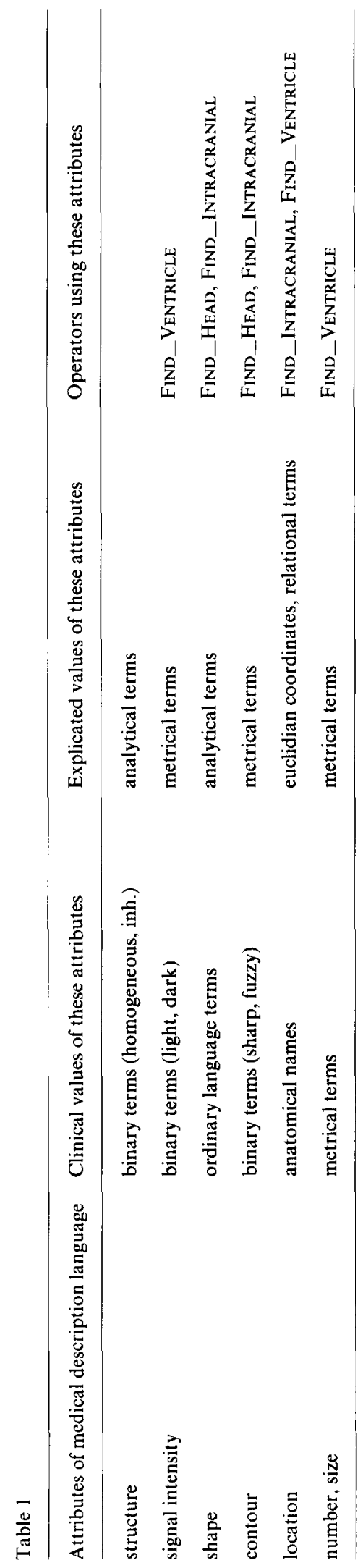


[7] Menhardt, W. and K.-H. Schmidt (1987). Wissensbasierte Konfigurierung von Interpretationsoperatoren anhand eines hierarchischen Szenenmodells. Proc. 9. DAGM-Symposion Braunschweig, Informatik-Fachberichte, Band 149 , Springer, Berlin, pp. 310-314.

[8] Neumann, B. (1986). Wissensbasierte Konfigurierung von Bildverarbeitungssystemen. Proc. 8, DAGM-Symposion Paderborn, Informatik-Fachberichte, Band 125, Springer, Berlin, pp. 206-218.

[9] Newton. T.H. and D.G. Potts, eds. (1983). Modern Neuroradiology, Vol. 2 (Advanced Imaging Techniques), Clavadel Press, San Anselmo, CA.

[10] Newell, J.A. (1986). Recognition of parts of anatomy using a symbolic structural model of a CT image of the brain. Proc. of the 2nd Intern. Conf. on Image Proc. and its Appl., London, June 1986.

[11] Ohlander, R.B. (1975). Analysis of natural scenes, PhD The- sis, Comp. Sci. Dept., Carnegie-Mellon Univ., Pittsburgh, PA.

[12] Schmidt, K.-H. (1988). Explikation medizinischer Beobachtungssprachen, Proc. Workshop 'Wissensarten und ihre Darstellung', Informatik-Fachberichte, Springer, Berlin, to appear.

[13] Stieh1, H.S. (1980). Automatische Verarbeitung und Analyse von kranialen Computertomogrammen, Dissertation, TU Berlin.

[14] Tenenbaum, J.M. and H.G. Barrow (1977). Experiments in interpretation guided segmentation. Artificial Intelligence 8 , 241274.

[15] Woods, W. (1975). What's in a link: foundations for semantic networks. In: Bobrow, D. and A. Collins, Eds., Representation and Understanding, Academic Press, New York. 